\title{
Faster Fully Homomorphic Encryption
}

\author{
Damien Stehlé ${ }^{1}$ and Ron Steinfeld ${ }^{2}$ \\ ${ }^{1}$ CNRS, Laboratoire LIP (U. Lyon, CNRS, ENS de Lyon, INRIA, UCBL), \\ 46 Allée d'Italie, 69364 Lyon Cedex 07, France \\ damien.stehle@gmail.com \\ http://perso.ens-lyon.fr/damien.stehle \\ ${ }^{2}$ Centre for Advanced Computing - Algorithms and Cryptography, \\ Department of Computing, Macquarie University, NSW 2109, Australia \\ ron.steinfeld@mq.edu.au \\ http://www.ics.mq.edu.au/ rons/
}

\begin{abstract}
We describe two improvements to Gentry's fully homomorphic scheme based on ideal lattices and its analysis: we provide a more aggressive analysis of one of the hardness assumptions (the one related to the Sparse Subset Sum Problem) and we introduce a probabilistic decryption algorithm that can be implemented with an algebraic circuit of low multiplicative degree. Combined together, these improvements lead to a faster fully homomorphic scheme, with a $\widetilde{O}\left(\lambda^{3.5}\right)$ bit complexity per elementary binary add/mult gate, where $\lambda$ is the security parameter. These improvements also apply to the fully homomorphic schemes of Smart and Vercauteren [PKC'2010] and van Dijk et al. [Eurocrypt'2010].
\end{abstract}

Keywords: fully homomorphic encryption, ideal lattices, SSSP.

\section{Introduction}

A homomorphic encryption scheme allows any party to publicly transform a collection of ciphertexts for some plaintexts $\pi_{1}, \ldots, \pi_{n}$ into a ciphertext for some function/circuit $f\left(\pi_{1}, \ldots, \pi_{n}\right)$ of the plaintexts, without the party knowing the plaintexts themselves. Such schemes are well known to be useful for constructing privacy-preserving protocols, for example as required in 'cloud computing' applications: a user can store encrypted data on a server, and allow the server to process the encrypted data without revealing the data to the server. For over 30 years, all known homomorphic encryption schemes supported only a limited set of functions $f$, which restricted their applicability. The theoretical problem of constructing a fully homomorphic encryption scheme supporting arbitrary functions $f$, was only recently solved by the breakthrough work of Gentry [9]. More recently, two further fully homomorphic schemes were presented [26 5], following Gentry's framework. The underlying tool behind all these schemes is the use of Euclidean lattices, which have previously proved powerful for devising many cryptographic primitives (see, e.g., 21] for a recent survey).

A central aspect of Gentry's fully homomorphic scheme (and the subsequent schemes) is the ciphertext refreshing Recrypt operation. The ciphertexts in 
Gentry's scheme contain a random 'noise' component that grows in size as the ciphertext is processed to homomorphically evaluate a function $f$ on its plaintext. Once the noise size in the ciphertext exceeds a certain threshold, the ciphertext can no longer be decrypted correctly. This limits the number of homomorphic operations that can be performed. To get around this limitation, the Recrypt operation allows to 'refresh' a ciphertext, i.e., given a ciphertext $\psi$ for some plaintext $\pi$, to compute a new ciphertext $\psi^{\prime}$ for $\pi$ (possibly for a different key), but such that the size of the noise in $\psi^{\prime}$ is smaller than the size of the noise in $\psi$. By periodically refreshing the ciphertext (e.g., after computing each gate in $f$ ), one can then evaluate arbitrarily large circuits $f$.

The Recrypt operation is implemented by evaluating the decryption circuit of the encryption scheme homomorphically, given 'fresh' (low noise) ciphertexts for the bits of the ciphertext to be refreshed and the scheme's secret key. This homomorphic computation of the decryption circuit must of course be possible without any ciphertext refreshing, a condition referred to as bootstrappability. Thus, the complexity (in particular circuit depth, or multiplicative degree) of the scheme's decryption circuit is of fundamental importance to the feasibility and complexity of the fully homomorphic scheme. Unfortunately, the relatively high complexity of the decryption circuit in the schemes 9265 , together with the tension between the bootstrappability condition and the security of the underlying hard problems, implies the need for large parameters and leads to resulting encryption schemes of high bit-complexity.

Our Contributions. We present improvements to Gentry's fully homomorphic scheme 9] and its analysis, that reduce its complexity. Overall, letting $\lambda$ be the security parameter (i.e., all known attacks against the scheme take time $\geq 2^{\lambda}$ ), we obtain a $\widetilde{O}\left(\lambda^{3.5}\right)$ bit complexity for refreshing a ciphertext corresponding to a 1-bit plaintext. This is the cost per gate of the fully homomorphic scheme. To compare with, Gentry [8, Ch. 12] claims a $\widetilde{O}\left(\lambda^{6}\right)$ bound, although the proof is incomplete 1 .

The improved complexity stems from two sources. First, we give a more aggressive security analysis of the Sparse Subset Sum Problem (SSSP) against lattice attacks, compared to the analysis given in 9]. The SSSP, along with the Ideal lattice Bounded Distance Decoding (BDD) problem, are the two hard problems underlying the security of Gentry's fully homomorphic scheme. In his security analysis of BDD, Gentry uses the best known complexity bound for the approximate shortest vector problem (SVP) in lattices, but in analyzing SSSP, Gentry assumes the availability of an exact SVP oracle. Our new finer analysis of SSSP takes into account the complexity of approximate SVP, making it more consistent with the assumption underlying the analysis of the BDD problem, and leads to smaller parameter choices. Second, we relax the definition of fully homomorphic encryption to allow for a negligible but non-zero probability

${ }^{1}$ This bound is claimed to hold for the scheme after Optimizations 1 and 2 of 8 , Se. 12.3], but the analysis does not include the cost of the ciphertext expansion nor details which decryption circuit is applied homomorphically. For instance, the decryption circuit from [5, Le. 6.3] is too costly to derive the bound. These gaps can be filled using Section 6.2 of the present article, and the bound $\widetilde{O}\left(\lambda^{6}\right)$ indeed holds. 
of decryption error. We then show that, thanks to the randomness underlying Gentry's 'SplitKey' key generation for his squashed decryption algorithm (i.e., the decryption algorithm of the bootstrappable scheme), if one allows a negligible decryption error probability, then the rounding precision used in representing the ciphertext components can be roughly halved, compared to the precision in [9] which guarantees zero error probability. The reduced ciphertext precision allows us to decrease the degree of the decryption circuit. We concentrate on Gentry's scheme [9], but our improvements apply equally well to the other related schemes [265].

Notation. Vectors will be denoted in bold. If $\boldsymbol{x} \in \mathbb{R}^{n}$, then $\|\boldsymbol{x}\|$ denotes the Euclidean norm of $\boldsymbol{x}$. We make use of the Landau notations $O(\cdot), \widetilde{O}(\cdot), \omega(\cdot), \Omega(\cdot)$, $\widetilde{\Omega}(\cdot), \Theta(\cdot), \widetilde{\Theta}(\cdot)$. If $n$ grows to infinity, we say that a function $f(n)$ is negligible if it is asymptotically $\leq n^{-c}$ for any $c>0$. If $X$ is a random variable, $E[X]$ denotes its mean and $\operatorname{Pr}[X=x]$ denotes the probability of the event " $X=x$ ". We say that a sequence of events $E_{n}$ holds with overwhelming probability if $\operatorname{Pr}\left[\neg E_{n}\right] \leq f(n)$ for a negligible function $f$. We will use the following variant of the Hoeffding bound [13].

Lemma 1.1. Let $X_{1}, \ldots, X_{t}$ denote independent random variables with mean $\mu$, where $X_{i} \in\left[a_{i}, b_{i}\right]$ for some $\boldsymbol{a}, \boldsymbol{b} \in \mathbb{R}^{t}$. Let $X=\sum_{i} X_{i}$. Then:

$$
\forall k \geq 0: \operatorname{Pr}[|X-t \mu| \geq k] \leq 2 \cdot \exp \left(-2 k^{2} /\|\boldsymbol{b}-\boldsymbol{a}\|^{2}\right) .
$$

Remark. Due to space limitations, some contents of the article are only given in the appendices of the full version, which is available on the authors' webpages. These include: a sketch of Gentry's bootstrapping transformation [9], adapted to handle decryption errors; a proof that an ideal sampled from Gentry's distribution [11] is of prime determinant with overwhelming probability, when the considered ring is $\mathbb{Z}[x] /\left(x^{2^{k}}+1\right)$; the proofs of Lemmata 3.2 and 3.3, and the application of our improvements to other fully homomorphic encryption schemes.

\section{Reminders}

For a detailed introduction to the computational aspects of lattices, we refer to [20]. The article [10] provides an intuitive description of Gentry's fully homomorphic scheme.

\subsection{Euclidean Lattices}

An $n$-dimensional lattice $L$ is the set of all integer linear combinations of some linearly independent vectors $\boldsymbol{b}_{1}, \ldots, \boldsymbol{b}_{n} \in \mathbb{Z}^{n}$, i.e., $L=\sum \mathbb{Z} \boldsymbol{b}_{i}$. The $\boldsymbol{b}_{i}$ 's are called a basis of $L$. A basis $B=\left(\boldsymbol{b}_{1}, \ldots, \boldsymbol{b}_{n}\right) \in \mathbb{Z}^{n \times n}$ is said to be in Hermite Normal Form (HNF) if $b_{i, j}=0$ for $i>j$ and $0 \leq b_{i, j}<b_{i, i}$ otherwise. The HNF of a lattice is unique and can be computed in polynomial time given any basis, which arguably makes it a worst-case basis [19]. To a basis $B=\left(\boldsymbol{b}_{1}, \ldots, \boldsymbol{b}_{n}\right) \in \mathbb{Z}^{n \times n}$ for lattice $L$, we associate the fundamental parallelepiped $\mathcal{P}(B)=\left\{\boldsymbol{v}=\sum_{i} y_{i} \cdot \boldsymbol{b}_{i}\right.$ : 
$\left.y_{i} \in(-1 / 2,1 / 2]\right\}$. For a vector $\boldsymbol{v} \in \mathbb{R}^{n}$, we denote by $\boldsymbol{v} \bmod B$ the unique vector $\boldsymbol{v}^{\prime} \in \mathcal{P}(B)$ such that $\boldsymbol{v}-\boldsymbol{v}^{\prime} \in L$. Note that $\boldsymbol{v}^{\prime}=\boldsymbol{v}-B\left\lfloor B^{-1} \boldsymbol{v}\right\rceil$, where $\lfloor\cdot\rceil$ rounds the coefficients to the nearest integers (upwards in case of a real that is equally distant to two consecutive integers).

The minimum $\lambda_{1}(L)$ is the norm of any shortest non-zero vector in $L$. More generally, the $i$ th minimum $\lambda_{i}(L)$ is the radius of the smallest ball containing $i$ linearly independent lattice vectors. We define the lattice amplitude as the ratio $\lambda_{n}(L) / \lambda_{1}(L)$. We now define two parametrized families of algorithmic problems that are central for Euclidean lattices. Let $\gamma \geq 1$ be a function of the dimension. The $\gamma$-SVP (for Shortest Vector Problem) computational problem consists in finding a vector $\boldsymbol{b} \in L$ such that $0<\|\boldsymbol{b}\| \leq \gamma \lambda_{1}(L)$, given as input an arbitrary basis for $L$. The $\gamma$-BDD (for Bounded Distance Decoding) computational problem consists in finding a vector $\boldsymbol{b} \in L$ closest to $\boldsymbol{t}$ given as inputs an arbitrary basis for $L$ and a target vector $\boldsymbol{t}$ whose distance to $L$ is $\leq \gamma^{-1} \lambda_{1}(L)$. Solving $\gamma$-SVP and $\gamma$-BDD are in general computationally hard problems. The best algorithms for solving them for $\gamma=1$ (1422]) run in time exponential with respect to the dimension. On the other hand, the smallest $\gamma$ one can achieve in polynomial time is exponential, up to poly-logarithmic factors in the exponent ([17 241]). For intermediate $\gamma$, the best strategy is the hierarchical reduction of [24], and leads to the following conjecture.

Lattice 'Rule of Thumb' Conjecture. There exist absolute constants $c_{1}, c_{2}>$ 1 such that for any $\lambda$ and any dimension $n$, for any $n$-dimensional lattice with amplitude $\leq \gamma / c_{2}$, one cannot solve $\gamma$-SVP (resp. $\gamma$-BDD) in time smaller than $2^{\lambda}$, with $\gamma=c_{1}^{n / \lambda}$.

Let us discuss the conjecture. One often considers the lattice gap $\frac{\lambda_{2}}{\lambda_{1}}$. If $\frac{\lambda_{2}}{\lambda_{1}}>$ $\gamma$, then $\gamma$-SVP is equivalent to $\gamma^{\prime}$-SVP for any $\gamma^{\prime}<\frac{\lambda_{2}}{\lambda_{1}}$ : a $\gamma^{\prime}$-SVP solver is guaranteed to output a multiple of a shortest vector, from which solving SVP is easy. Similarly, if $\frac{\lambda_{2}}{\lambda_{1}}=O(1)$ but $\frac{\lambda_{3}}{\lambda_{2}}>\gamma$, then lattice reduction will return a basis whose first two vectors span a sublattice containing vectors reaching $\lambda_{1}$ and $\lambda_{2}$ : SVP can then be solved by 2 -dimensional reduction. This explains why we consider $\frac{\lambda_{n}}{\lambda_{1}}$ rather than the more standard $\frac{\lambda_{2}}{\lambda_{1}}$. Note that for most common lattices, there is no a priori reason to expect $\lambda_{n}$ to be significantly larger than $\lambda_{2}$. Finally, when $\frac{\lambda_{n}}{\lambda_{1}} \leq \gamma$, the complexity of $\gamma$-SVP does not seem to depend on $\frac{\lambda_{n}}{\lambda_{1}}$. The experimental results in [7] seem to be consistent with this conjecture.

Algorithmic improvements have been proposed (e.g., [6 16]), but they have only led to better constants, without changing the overall framework. The conjecture seems to hold even if one considers quantum computers [18. We will consider it for two families of lattices: no algorithm is known to perform nonnegligibly better for them than for general lattices.

For a lattice $L$, we $\operatorname{define} \operatorname{det} L$ as $|\operatorname{det} B|$ for any basis $B$. Minkowski's theorem provides a link between the minimum and the determinant.

Theorem 2.1 ([4, III.2.2]). Let $L$ be an $n$-dimensional lattice and $V$ be a compact convex set that is symmetric about the origin. Let $m \geq 1$ be an integer. If $\operatorname{vol}(V)$ $\geq m 2^{n} \operatorname{det}(L)$, then $V$ contains at least $m$ non-zero pairs of points $\pm \mathbf{b}$ of $L$. 


\section{$2.2 \quad$ Ideal Lattices}

Let $f \in \mathbb{Z}[x]$ a monic degree $n$ irreducible polynomial. Let $R$ denote the polynomial ring $\mathbb{Z}[x] / f$. Let $I$ be an (integral) ideal of $R$, i.e., a subset of $R$ that is closed under addition, and multiplication by arbitrary elements of $R$. By mapping polynomials to the vectors of their coefficients, we see that the ideal $I$ corresponds to a sublattice of $\mathbb{Z}^{n}$ : we can thus view $I$ as both a lattice and an ideal. An ideal lattice for $f$ is a sublattice of $\mathbb{Z}^{n}$ that corresponds to an ideal $I \subseteq \mathbb{Z}[x] / f$. In the following, an ideal lattice will implicitly refer to an $f$-ideal lattice. For $v \in R$ we denote by $\|v\|$ its Euclidean norm (as a vector). We define a multiplicative expansion factor $\gamma_{\times}(R)$ for the ring $R$ by $\gamma_{\times}(R)=\max _{u, v \in R} \frac{\|u \times v\|}{\|u\| \cdot\|v\|}$. A typical choice is $f=x^{n}+1$ with $n$ a power of 2 , for which $\gamma_{\times}(R)=\sqrt{n}$ (see [9, Th. 9]).

Two ideals $I$ and $J$ of $R$ are said coprime if $I+J=R$, where $I+J=\{i+j$ : $i \in I, j \in J\}$. An ideal $I$ is said prime of degree 1 if $\operatorname{det}(I)$ is prime. For an ideal $J$ of $R$, we define $J^{-1}=\{\boldsymbol{v} \in \mathbb{Q}[x] / f: \forall \boldsymbol{u} \in J, \boldsymbol{u} \times \boldsymbol{v} \in R\}$. This is a fractional ideal of $R$, and $J^{-1} \subseteq \frac{1}{\operatorname{det} J} R\left(\right.$ since $(\operatorname{det} J) \cdot R \subseteq J$ ). If $f=x^{n}+1$ with $n$ a power of 2 , then $R$ is the ring of integers of the $(2 n)$ th cyclotomic field and $J^{-1} \times J=R$ for any integral ideal $J$ (the product of two ideals $I_{1}$ and $I_{2}$ being the ideal generated by all products $i_{1} \cdot i_{2}$ with $i_{1} \in I_{1}$ and $i_{2} \in I_{2}$ ). An ideal $I$ is said principal if it is generated by a single element $r \in I$, and then we write $I=(r)$. We define $\operatorname{rot}_{f}(r) \in \mathbb{Q}^{n \times n}$ as the basis of $I$ consisting of the $x^{k} r(x) \bmod f$ 's, for $k \in[0, n-1]$.

If $I$ is an ideal lattice for $f=x^{n}+1$, then we have $\lambda_{1}(I) \geq \operatorname{det}(I)^{1 / n}$ : an easy way to prove it is to notice that the rotations $x^{k} v$ of any shortest non-zero vector $v$ form a basis of a full-rank sublattice of $I$, and to use the inequalities $\lambda_{1}(I)^{n}=\prod_{k}\left\|x^{k} v\right\| \geq \operatorname{det}((v)) \geq \operatorname{det} I$.

\subsection{Homomorphic Encryption}

In this section, we review definitions related to homomorphic encryption. Our definitions are based on [98, but we slightly relax the definition of decryption correctness, to allow a negligible probability of error. This is crucial for our probabilistic improvement to Gentry's Recrypt algorithm.

Definition 2.1. A homomorphic encryption scheme Hom consists of four algorithms:

- KeyGen: Given security parameter $\lambda$, returns a secret key sk and a public key $p k$.

- Enc: Given plaintext $\pi \in\{0,1\}$ and public key pk, returns ciphertext $\psi$.

- Dec: Given ciphertext $\psi$ and secret key sk, returns plaintext $\pi$.

- Eval: Given public key pk, a t-input circuit $C$ (consisting of addition and multiplication gates modulo 2), and a tuple of ciphertexts $\left(\psi_{1}, \ldots, \psi_{t}\right)$ (corresponding to the $t$ input bits of $C$ ), returns a ciphertext $\psi$ (corresponding to the output bit of $C$ ). 
Hom is said correct for a family $\mathcal{C}$ of circuits with $\leq t=\mathcal{P}$ oly $(\lambda)$ input bits if for any $C \in \mathcal{C}$ and input bits $\left(\pi_{i}\right)_{i \leq t}$, the following holds with overwhelming probability over the randomness of KeyGen and Enc:

$$
\operatorname{Dec}\left(s k, \operatorname{Eval}\left(p k, C,\left(\psi_{1}, \ldots, \psi_{t}\right)\right)\right)=C\left(\pi_{1}, \ldots, \pi_{t}\right),
$$

where $(s k, p k)=\operatorname{KeyGen}(\lambda)$ and $\psi_{i}=\operatorname{Enc}\left(p k, \pi_{i}\right)$ for $i=1, \ldots, t$.

Hom is said compact if for any circuit $C$ with $\leq t=\mathcal{P}$ oly $(\lambda)$ input bits, the bit-size of the ciphertext $\operatorname{Eval}\left(p k, C,\left(\psi_{1}, \ldots, \psi_{t}\right)\right)$ is bounded by a fixed polynomial $b(\lambda)$.

Gentry [9] defined the powerful notion of a bootstrappable homomorphic encryption scheme: one that can homomorphically evaluate a decryption of two ciphertexts followed by one gate applied to the decrypted values. We also relax this notion to allow decryption errors.

Definition 2.2. Let Hom = (KeyGen, Enc, Dec, Eval) denote a homomorphic encryption scheme. We define two circuits:

- Dec-Add: Takes as inputs a secret key sk and two ciphertexts $\psi_{1}, \psi_{2}$, and computes $\operatorname{Dec}\left(s k, \psi_{1}\right)+\operatorname{Dec}\left(s k, \psi_{2}\right) \bmod 2$.

- Dec-Mult: Takes as inputs a secret key sk and two ciphertexts $\psi_{1}, \psi_{2}$, and computes $\operatorname{Dec}\left(s k, \psi_{1}\right) \times \operatorname{Dec}\left(s k, \psi_{2}\right) \bmod 2$.

Hom is said bootstrappable if it is correct for Dec-Add and Dec-Mult.

Gentry discovered that a bootstrappable homomorphic encryption can be used to homomorphically evaluate arbitrary circuits. More precisely, he proved the following result (adapted to allow for decryption error). The construction is sketched in the full version.

Theorem 2.2 ([9, Se. 2]). Given a bootstrappable homomorphic encryption scheme Hom, and parameter $d=\mathcal{P}$ oly $(\lambda)$, it is possible to construct another homomorphic encryption scheme $\mathrm{Hom}^{(d)}$ that is compact and correct for all circuits of size Poly $(\lambda)$. Furthermore, if the scheme Hom is semantically secure, then so is the scheme $\mathrm{Hom}^{(d)}$.

\section{Summary of Gentry's Fully Homomorphic Scheme}

We now review Gentry's fully homomorphic encryption scheme [9].

\subsection{The Somewhat Homomorphic Scheme}

We first recall Gentry's somewhat homomorphic encryption scheme (see [8, Se. 5.2 and Ch. 7]) which supports a limited number of multiplications. It is the basis for the bootstrappable scheme presented in Subsection 3.3. The scheme, described in Figure 1 produces ciphertexts in the ring $R=\mathbb{Z}[x] / f$ for a suitable 
irreducible degree $n$ monic polynomial $f$. In this paper, we will assume $f=x^{n}+1$ with $n$ a power of 2 . Here $n$ is a function of the security parameter $\lambda$.

The key generation procedure generates two coprime ideals $I$ and $J$ of $R$. The ideal $I$ has basis $B_{I}$. To simplify the scheme (and optimize its efficiency), a convenient choice, which we assume in this paper, is to take $I=(2)$ : Reduction of $v$ modulo $I$ corresponds to reducing the coefficients of the vector/polynomial $v$ modulo 2. The ideal $J$ is generated by an algorithm IdealGen, that given $(\lambda, n)$, generates a 'good' secret basis $B_{J}^{s k}$ (consisting of short, nearly orthogonal vectors) and computes its HNF to obtain a 'bad' public basis $B_{J}^{p k}$. Suggestions for concrete implementations of IdealGen are given in [8, Se. 7.6], [11] and [26]. To obtain the $\widetilde{O}\left(\lambda^{3.5}\right)$ bit complexity bound, we will assume that $J$ is a degree 1 prime ideal, which is the case with the implementation of [26] and is also the case with probability exponentially close to 1 for the distribution considered in [1] (see full version). Associated with IdealGen is a parameter $r_{D e c}$, which is a lower bound on the radius of the largest origin-centered ball which is contained inside $\mathcal{P}\left(B_{J}^{s k}\right)$. In all cases we have $r_{D e c} \geq \lambda_{1}(J) / \mathcal{P}$ oly $(n)$ (see, e.g., [8, Le. 7.6.2]). Using Babai's rounding-off algorithm [1] with $B_{J}^{s k}$, the decryptor can recover the point of $J$ closest to any target vector within distance $r_{D e c}$ of $J$ (see [8, Le. 7.6.1]).

- $\operatorname{KeyGen}(\lambda)$ : Run IdeaIGen $(\lambda, n)$ to generate secret/public bases $\left(B_{J}^{s k}, B_{J}^{p k}\right)$ for ideal $J$ such that $\mathcal{P}\left(B_{J}^{s k}\right)$ contains an origin-centered ball of radius $r_{D e c} \approx \lambda_{1}(J)$. Return public key $p k=B_{J}^{p k}$ and secret key $s k=B_{J}^{s k}$.

- $\operatorname{Enc}(p k, \pi)$ : Given plaintext $\pi \in\{0,1\}$ and public key $p k, \operatorname{run} \operatorname{Samp}(I, \pi)$ to get $\pi^{\prime} \in \pi+I$ with $\left\|\pi^{\prime}\right\| \leq r_{E n c}$. Return ciphertext $\psi=\pi^{\prime} \bmod B_{J}^{p k}$.

- $\operatorname{Dec}(s k, \psi)$ : Given ciphertext $\psi$ and secret key $s k$, returns $\pi=\left(\psi \bmod B_{J}^{s k}\right) \bmod I$.

- $\operatorname{Eval}\left(p k, C,\left(\psi_{1}, \ldots, \psi_{t}\right)\right)$ : Given public key $p k$, circuit $C$ and ciphertexts $\psi_{1}, \ldots, \psi_{t}$, for each add or multiply gate in $C$, perform a + or $\times$ operation in $R \bmod B_{J}^{p k}$, respectively, on the corresponding ciphertexts. Return the ciphertext $\psi$ corresponding to the output of $C$.

Fig. 1. Gentry's Somewhat Homomorphic Encryption Scheme SomHom

The plaintext space is a subset of $\mathcal{P}(I)$, that we assume to be $\{0,1\}$. The encryption algorithm uses a sampling algorithm Samp, which given $\left(B_{I}, \boldsymbol{x}\right)$ for a vector $x \in R$, samples a 'short' vector in the coset $x+I$. Concrete implementations of Samp are given in [8, Se. 7.5 and 14.1]. Associated with Samp is a parameter $r_{E n c}$, which is a (possibly probabilistic) bound on the norms of vectors output by Samp. For both implementations, one can set $r_{\text {Enc }}=\mathcal{P}$ oly $(n)$. To encrypt a message $\pi$, a sample $\pi+i$ from the coset $\pi+I$ is generated, and the result is reduced modulo the public basis $B_{J}^{p k}: \psi=\pi+i \bmod B_{J}^{p k}$. It is assumed that $r_{E n c}<r_{D e c}$. Therefore, by reducing $\psi$ modulo the secret basis $B_{J}^{s k}$ one can recover $\pi+i$, and then plaintext $\pi$ can be recovered by reducing modulo $B_{I}$.

Homomorphic addition and multiplication of the encrypted plaintexts $\pi_{1}, \pi_{2}$ modulo $B_{I}$ are supported by performing addition and multiplication respectively in the $\operatorname{ring} R$ on the corresponding ciphertexts modulo $B_{J}^{p k}$. Namely, 
for $\psi_{1}=\pi_{1}+i_{1} \bmod B_{J}^{p k}, \psi_{2}=\pi_{2}+i_{2} \bmod B_{J}^{p k}$ with $i_{1}, i_{2} \in I$, we have $\psi_{1}+\psi_{2} \bmod B_{J}^{p k} \in\left(\pi_{1}+\pi_{2}\right)+I$ and $\psi_{1} \times \psi_{2} \bmod B_{J}^{p k} \in\left(\pi_{1} \times \pi_{2}\right)+I \bmod B_{J}^{p k}$. However, for ensuring correct decryption of these new ciphertexts, we need that $\left\|\left(\pi_{1}+i_{1}\right)+\left(\pi_{2}+i_{2}\right)\right\|,\left\|\left(\pi_{1}+i_{1}\right) \times\left(\pi_{2}+i_{2}\right)\right\| \leq r_{D e c}$. This limits the degree of polynomials that can be evaluated homomorphically. Note that our choice for $J$ implies that a ciphertext reduced modulo $B_{J}^{p k}$ is simply an integer modulo $\operatorname{det}(J)$ and thus homomorphic evaluations modulo $B_{J}^{p k}$ reduce to integer arithmetic modulo $\operatorname{det}(J)$ (such as in [26]).

\subsection{A Tweaked Somewhat Homomorphic Scheme}

Gentry [8, Ch. 8] introduced tweaks to SomHom to simplify the decryption algorithm towards constructing a fully homomorphic scheme. The tweaked scheme SomHom' differs from the original scheme in the key generation and decryption algorithm, as detailed in Figure 2 .

- KeyGen' $(\lambda)$ : Run KeyGen $(\lambda)$ to obtain $\left(B_{J}^{s k}, B_{J}^{p k}\right)$. From $B_{J}^{s k}$, compute a vector $\boldsymbol{v}_{J}^{s k} \in J^{-1}$ such that $\mathcal{P}\left(\operatorname{rot}_{f}\left(\boldsymbol{v}_{J}^{s k}\right)^{-1}\right)$ contains a ball of radius $r_{D e c}^{\prime}=\frac{r_{D e c}}{8 \sqrt{2} n^{2.5}}$ (see [8, Le. 8.3.1]). Return public key $p k=B_{J}^{p k}$ and secret key $s k=B_{J}^{s k}$.

- $\operatorname{Dec}^{\prime}(s k, \psi)$ : Given ciphertext $\psi$ and secret key $s k$, return $\pi=\psi-\left\lfloor\boldsymbol{v}_{J}^{s k} \times \psi\right\rceil \bmod I$.

Fig. 2. Algorithms of the Tweaked Somewhat Homomorphic Encryption Scheme SomHom' that differ from those of SomHom

Gentry showed the following on the correctness of Dec'.

Lemma 3.1 (Adapted from [8, Le. 8.3.1 and 8.4.2]). A ciphertext $\psi=$ $\pi+i \bmod B_{J}^{p k}$ with $\|\pi+i\| \leq r_{\text {Dec }}^{\prime}$ is correctly decrypted to $\pi$ by Dec'. Moreover, if $\|\pi+i\| \leq r_{\text {Dec }}^{\prime}$, then each coefficient of $\boldsymbol{v}_{J}^{s k} \times \psi$ is within $1 / 8$ of an integer.

Let $C$ be a mod 2 circuit consisting of add and multiply gates with two inputs and one output. We let $g(C)$ denote the generalized circuit obtained from $C$ by replacing the add and multiply gates mod 2 by the + and $\times$ operations of the ring $R$, respectively. We say that circuit $C$ is permitted, if for any set of inputs $\boldsymbol{x}_{1}, \ldots, \boldsymbol{x}_{t}$ to $g(C)$ with $\left\|\boldsymbol{x}_{k}\right\| \leq r_{E n c}$ for $k=1, \ldots, t$, we have $\left\|g(C)\left(\boldsymbol{x}_{1}, \ldots, \boldsymbol{x}_{t}\right)\right\| \leq r_{D e c}^{\prime}$. A permitted circuit which is evaluated homomorphically on encryptions of plaintexts $\pi_{1}, \ldots, \pi_{t}$ will yield a ciphertext $\psi=g(C)\left(\pi_{1}+i_{1}, \ldots, \pi_{t}+i_{t}\right) \bmod B_{J}^{p k}$ that correctly decrypts to $C\left(\pi_{1}, \ldots, \pi_{t}\right)$, and such that the coefficients of $\boldsymbol{v}_{J}^{s k} \times \psi$ are within $1 / 8$ of an integer. As in [5, Le 3.4], we characterize the permitted circuits by the maximal degree of the polynomial evaluated by the circuit. Note that Gentry [98] considers the circuit depth, which is less flexible.

Lemma 3.2. Let $C$ be a mod 2 circuit, and $g(C)$ denote the corresponding generalized circuit over $R$, evaluating $h \in \mathbb{Z}\left[x_{1}, \ldots, x_{t}\right]$ of (total) degree $d$. The circuit $C$ is permitted if $\gamma_{\times}^{d-1}\|h\|_{1} r_{E n c}^{d} \leq r_{\text {Dec }}^{\prime}$. In particular, assuming that $h$ has coefficients in $\{0,1\}$, the circuit $C$ is permitted if $d$ satisfies 


$$
d \leq \frac{\log r_{D e c}^{\prime}}{\log \left(r_{E n c} \cdot \gamma_{\times} \cdot(t+1)\right)} .
$$

Remark. The polynomial $h$ referred to above is the one evaluated by the generalized circuit $g(C)$. For arbitrary circuits $C \bmod 2$, the polynomial $h$ may differ from the polynomial $h^{\prime}$ evaluated by the circuit $C \bmod 2$; in particular, the polynomial $h$ may have non-binary integer coefficients, and some may be multiples of 2 . However, for circuits $C$ for which $h$ has binary coefficients (the condition in the lemma), we have $h=h^{\prime}$ (this condition on $h$ is also needed, but is not explicitly stated in [5]).

\subsection{Gentry's Squashed Bootstrappable Scheme}

To make it bootstrappable, Gentry [8, Ch. 10] modified SomHom' by 'squashing' the decryption circuit. He moved some of the decryption computation to the encryption stage, by providing additional information in the public key. This results in the bootstrappable scheme SqHom described in Figure 3 The scheme introduces three new integer parameters $\left(p, \gamma_{s e t}, \gamma_{s u b}\right)$. Note that we incorporated Optimization 2 from [8, Ch. 12], which is made possible thanks to the choice $I=(2)$.

- KeyGen" $(\lambda)$ :

- Run KeyGen' to get $B_{J}^{p k}$ and $\boldsymbol{v}_{J}^{s k}$.

- Generate a uniform $\gamma_{s e t}$-bit vector $s=\left(s_{1}, \ldots, s_{\gamma_{s e t}}\right)$ with Hamming weight $\gamma_{s u b}$ and $s_{\gamma_{s e t}}=1$.

- Generate $\boldsymbol{t}_{1}, \ldots, \boldsymbol{t}_{\gamma_{\text {set }}-1}$ uniformly and independently from $J^{-1} \bmod B_{I}$. Compute $\boldsymbol{t}_{\gamma_{s e t}}=\boldsymbol{v}_{J}^{s k}-\sum_{k<\gamma_{s e t}} s_{k} \boldsymbol{t}_{k}$.

- Return $s k=s$ and $p k=\left(B_{J}^{p k} ; \boldsymbol{t}_{1}, \ldots, \boldsymbol{t}_{\gamma_{s e t}}\right)$.

- $\operatorname{Enc}^{\prime \prime}(p k, \pi)$ : Run Enc of SomHom' to generate ciphertext $\psi$. For $k=1, \ldots, \gamma_{s e t}$, compute $c_{k}$ on $p+1$ bits ( 1 bit before the binary point, and $p$ bits after) such that $\left|c_{k}-\left[\boldsymbol{t}_{k} \times \boldsymbol{\psi}\right]_{0} \bmod 2\right| \leq 2^{-p}$, where $[g]_{0}$ denotes the constant coefficient of the polynomial $g \in R$. Return ciphertext $\left(\psi ; c_{1}, \ldots, c_{\gamma_{s e t}}\right)$.

- $\operatorname{Dec}^{\prime \prime}\left(s k,\left(\psi ; c_{1}, \ldots, c_{\gamma_{s e t}}\right)\right)$ : Given expanded ciphertext $\left(\psi ; c_{1}, \ldots, c_{\gamma_{s e t}}\right)$ and secret key $s k$, return $\pi=[\psi]_{0}-\left\lfloor\sum_{k} s_{k} c_{k}\right\rceil \bmod 2$.

- Eval': Same as for SomHom' (while recomputing the $c_{k}$ 's, like in algorithm Enc' ${ }^{\prime \prime}$ ).

Fig. 3. Algorithms of the Squashed Scheme SqHom

Note that $\sum_{k} s_{k} c_{k} \approx \sum_{k} s_{k}\left[\boldsymbol{t}_{k} \times \boldsymbol{\psi}\right]_{0}=\left(\left[\left(\sum_{k} s_{k} \boldsymbol{t}_{k}\right) \times \boldsymbol{\psi}\right]_{0}\right)=\left[\boldsymbol{v}_{J}^{s k} \times \boldsymbol{\psi}\right]_{0}$, modulo 2. Hence, in terms of decryption correctness, SqHom differs from SomHom' only due to the rounding errors. The following lemma provides a sufficient precision $p$ (see also [5. Le. 6.1]). In Section [5] we will show that $p$ can be almost halved, using a probabilistic error analysis.

Lemma 3.3. If $p \geq 3+\log _{2} \gamma_{s u b}$, a ciphertext $\left(\psi ; c_{1}, \ldots, c_{\gamma_{\text {set }}}\right)$ of SqHom with $\psi=\pi+i \bmod B_{J}^{p k}$ and $\|\pi+i\| \leq r_{\text {Dec }}^{\prime}$ is correctly decrypted by the decryption algorithm Dec', and $\sum_{k} s_{k} c_{k}$ is within $1 / 4$ of an integer. 
For bootstrappability, we need to be able to implement the augmented decryption circuits Dec-Add and Dec-Mult with circuit degrees smaller than the degree capacity of the scheme. This is summarized in the following, in terms of the size $\gamma_{s u b}$ of the hidden subset in the secret key.

Theorem 3.1 (Adapted from [5, Th. 6.2]). Assuming that $\sum_{k} s_{k} c_{k}$ is within $1 / 4$ of an integer, the augmented decryption circuits Dec-Add and DecMult for scheme SqHom with precision parameter $p$ can be evaluated by circuits of degrees $\leq \gamma_{s u b} \cdot 2^{9} p^{1.71}$.

Proof. To decrypt $\psi$, we have to compute $\pi=[\psi]_{0}-\left\lfloor\sum_{k} s_{k} c_{k}\right\rceil \bmod 2$. We proceed as follows:

1- Compute $a_{k}=s_{k} \cdot c_{k}$ for $k=1, \ldots, \gamma_{s e t}$.

2- Let $a_{k, 0} . a_{k, 1} \ldots a_{k, p}$ be the bit representation of $a_{k}$. To sum the $a_{k}$ 's:

2.1- For $j=0, \ldots, p$, compute $W_{j}$, the Hamming weight of the bit vector $\left(a_{0, j}, \ldots, a_{\gamma_{s e t}, j}\right)$.

2.2- Compute $\pi=[\psi]_{0}-\sum_{j \leq p} W_{j} \cdot 2^{-j} \bmod 2$.

Note that because only $\gamma_{s u b}$ of the $a_{k}$ 's are non-zero, each Hamming weight $W_{j}$ is at most $\gamma_{s u b}$ and hence its binary representation has at most $\left\lceil\log _{2}\left(\gamma_{s u b}+1\right)\right\rceil$ bits. Step 1 requires a single multiplication mod 2 for each output bit, hence has degree 2. For Step 2.1, we use the following.

Lemma 3.4 (Adapted from [5, Le. 6.3]). Let $\left(\sigma_{1}, \ldots, \sigma_{t}\right)$ be a binary vector, and $W=W_{n} \ldots W_{0}$ be the binary representation of its Hamming weight. Then for any $k$, the bit $W_{k}$ can be expressed as a the evaluation in the $\sigma_{j}$ 's of an integer polynomial of degree exactly $2^{k}$.

We conclude that Step 2.1 can be computed by a circuit of degree $2^{\left\lceil\log _{2}\left(\gamma_{s u b}+1\right)\right\rceil} \leq$ $2 \gamma_{s u b}$. Using the ' 3 -for-2' trick [15], van Dijk et al. [5] show that Step 2.2 can be done with a circuit of degree $\leq 2^{\left\lceil\log _{3 / 2}(p+1)\right\rceil+4} \leq 2^{6} p^{1.71}$. The total degree of the decryption circuit is thus $\leq \gamma_{s u b} \cdot 2^{8} p^{1.71}$, and hence that of Dec-Add (resp. DecMult) is $\leq \gamma_{s u b} \cdot 2^{9} p^{1.71}$.

Combining Theorem 3.1 with Lemmata 3.2 and 3.3 , we get:

Corollary 3.1. The scheme SqHom is bootstrappable as long as

$$
\gamma_{s u b} \cdot 2^{9} \log ^{1.71}\left(\gamma_{s u b}+4\right) \leq \frac{\log r_{D e c}^{\prime}}{\log \left(r_{E n c} \cdot \gamma_{\times} \cdot(t+1)\right)} .
$$

\section{A Less Pessimistic Hardness Analysis of the SSSP}

The semantic security of Gentry's schemes SomHom and SomHom' relies on the hardness of a bounded distance decoding problem. As explained in Section 2, this hardness assumption is asymptotically well understood (with the lattice reduction 'rule of thumb' conjecture). When converted into the bootstrappable 
scheme SqHom, another hardness assumption is added, namely that of the so-called SplitKey distinguishing problem. To be precise, a semantic attack against SqHom either leads to an efficient ideal lattice BDD algorithm or to an efficient algorithm for the SplitKey distinguishing problem (see [9, Th. 10]). In [9. Th. 11.1.3], the following Sparse Vector Subset Sum Problem (SVSSP) is shown to reduce to the SplitKey distinguishing problem.

Definition 4.1 (SVSSP $\left.{ }_{\gamma_{s u b}, \gamma_{s e t}}\right)$. Let $\gamma_{s u b}$ and $\gamma_{\text {set }}$ be functions of the hardness parameter $\lambda$. Let $J$ be as generated by KeyGen, and $B_{I J}$ be the HNF of ideal IJ. The decisional SVSSP is as follows: Distinguish between $\left(\boldsymbol{a}_{1}, \ldots, \boldsymbol{a}_{\gamma_{\text {set }}}\right)$ chosen uniformly in $R \cap \mathcal{P}\left(B_{I J}\right)$ and the same but conditioned on the existence of a vector $\boldsymbol{s} \in\{0,1\} \gamma^{\gamma_{\text {set }}}$ of Hamming weight $\gamma_{\text {sub }}$ with $\sum_{k} s_{k} \boldsymbol{a}_{k}=0 \bmod I J$.

For our choice $I=(2)$, we have $B_{I J}=2 B_{J}^{p k}$, where $B_{J}^{p k}$ is the HNF of $J$. In the following, we use $q=\operatorname{det}\left(B_{I J}\right)=2^{n} \operatorname{det}(J)$. A simple birthday paradox attack runs in time $\approx\left(\begin{array}{c}\gamma_{s e t} \\ \gamma_{s u b}\end{array}\right)^{1 / 2}$. To achieve $2^{\lambda}$ hardness, we require that $\gamma_{s u b}=\Omega(\lambda)$ and $\gamma_{\text {set }} \geq 2 \gamma_{\text {sub }}$. We now analyze another attack, based on lattice reduction. Consider the lattice

$$
L=\left\{\boldsymbol{x} \in \mathbb{Z}^{\gamma_{s e t}}: \sum_{k \leq \gamma_{s e t}} x_{k} \cdot \boldsymbol{a}_{k}=0 \bmod I J\right\} .
$$

Since $q \mathbb{Z}^{\gamma_{\text {set }}} \subseteq L$, we have $\operatorname{dim} L=\gamma_{\text {set }}$. Furthermore, we have $\operatorname{det} L=$ $\left|\mathbb{Z}^{\gamma_{\text {set }}} / L\right|=\left|\phi\left(\mathbb{Z}^{\gamma_{\text {set }}}\right)\right| \leq \operatorname{det}\left(B_{I J}\right)=q$, where $\phi: \mathbb{Z}^{\gamma_{\text {set }}} \rightarrow \mathbb{Z}^{n} / I J$ is the map $\boldsymbol{x} \mapsto \sum_{k} x_{k} \boldsymbol{a}_{k} \bmod I J$. Also, the existence of the solution vector $\boldsymbol{s}$ implies that $1 \leq \lambda_{1}(L) \leq \sqrt{\gamma_{s u b}}$.

Suppose we are limited to a computational power of $2^{\lambda}$. The lattice reduction 'rule of thumb' conjecture suggests that we cannot find vectors in $L$ of norms $\leq$ $U:=c_{1}^{\frac{\gamma_{s e t}}{\lambda}}$, assuming that $\frac{\lambda_{\gamma_{s e t}}(L)}{\lambda_{1}(L)} \leq U / c_{2}$. Apart from the unusual smallness of the lattice minimum, there is no reason to expect the remaining $\lambda_{i}(L)$ 's to vary significantly: the lattice gap $\frac{\lambda_{2}(L)}{\lambda_{1}(L)}$ and the lattice amplitude $\frac{\lambda_{\gamma_{\text {set }}}(L)}{\lambda_{1}(L)}$ should be similar. Now, there are $\leq m:=U \sqrt{\gamma_{s u b}}$ pairs of non-zero multiples $\pm k \cdot \mathbf{s}$ with norm $\leq U \cdot \lambda_{1}(L) \leq U \sqrt{\gamma_{s u b}}$. At the same time, Minkowski's theorem (Theorem 2.1) asserts that there are far more lattice vectors of norm $\leq U / c_{2}$.

Lemma 4.1. Assuming that $\frac{\pi^{\frac{\gamma_{s e t}}{2}}}{\Gamma\left(\frac{\gamma_{s e t}+2}{2}\right)} \cdot\left(U / c_{2}\right)^{\gamma_{\text {set }}} \geq\left(2^{\lambda} m\right) \cdot 2^{\gamma_{\text {set }}} \cdot q$, we have $\left|L \cap \mathcal{B}\left(\mathbf{0}, U / c_{2}\right)\right| \geq 2^{\lambda} m$.

Note that if the condition in Lemma 4.1 holds, then for any $\lambda \geq 1$, the ball of radius $U \lambda_{1}(L) \geq U / c_{2}$ contains more than $m$ pairs of non-zero points of $L$, so the lattice gap $\frac{\bar{\lambda}_{2}(L)}{\lambda_{1}(L)}$ must be $\leq U / c_{2}$.

It seems reasonable to assume that the lattice points that are not multiples of $\mathbf{s}$ do not provide information towards solving SVSSP. Also, we heuristically expect lattice reduction to return one of these relevant vectors with probability $\approx 2^{-\lambda}$ if they constitute a fraction $2^{-\lambda}$ of the total number of lattice vectors of norm 
$\leq U$. Under these assumptions, if the computational effort of lattice reduction is limited to $2^{\lambda}$ and if we wish to bound the likelihood of finding a relevant vector by $2^{-\lambda}$, it seems sufficient to set the parameters so that:

$$
c_{1}^{\frac{\gamma_{s e t}^{2}}{\lambda}} \geq 2^{\lambda} \cdot \gamma_{\text {set }}^{\Omega\left(\gamma_{s e t}\right)} \cdot q
$$

As $\gamma_{\text {set }}=\widetilde{\Omega}(\lambda)$, the above is implied by $\frac{\gamma_{\text {set }}^{2}}{\lambda}=\widetilde{\Omega}(\log q)$. Note that this condition is less restrictive than the corresponding one used in [9265] (i.e., $\left.\gamma_{\text {set }}=\Omega(\log q)\right)$.

Remark. In algorithm KeyGen", the SVSSP instances satisfy $s_{\gamma_{s e t}}=1$. This does not result in any security reduction, as an attacker can guess an $i$ such that $s_{i}=1$ and then permute indices $i$ and $\gamma_{\text {set }}$.

Remark. Our analysis differs in two ways from the one from [9] relying on [23]: for consistency with the hardness analysis of the ideal BDD, we consider an approximate SVP solver rather than an exact SVP solver; secondly, we do not consider the 'replay' attack from [23] (which would lead to larger involved constants), as contrarily to the case of server-aided RSA, only one instance of the SSSP is given.

\section{Improved Ciphertext Refreshing Algorithm}

As explained in the proof of Theorem 3.1, the main component in the degree of the decryption algorithm comes from the addition of the rationals $s_{k} c_{k}=$ $\left[s_{k} \boldsymbol{t}_{k} \times \boldsymbol{\psi}\right]_{0} \bmod 2$. This accounts for degree $\gamma_{s u b}$, and all other components of degree are negligible compared to this one.

Recall that $\boldsymbol{t}_{1}, \ldots, \boldsymbol{t}_{\gamma_{s e t}-1}$, and hence also $\left[\boldsymbol{t}_{1} \times \boldsymbol{\psi}\right]_{0} \bmod 2, \ldots,\left[\boldsymbol{t}_{\gamma_{s e t}-1} \times\right.$ $\boldsymbol{\psi}]_{0} \bmod 2$ 's are chosen independently with identical distribution (iid), and that $\boldsymbol{t}_{\gamma_{s e t}}=\boldsymbol{v}_{J}^{s k}-\sum_{k<\gamma_{s e t}} s_{k} \boldsymbol{t}_{k} \bmod 2$. We are to exploit the iid-ness of the first $\boldsymbol{t}_{i}$ 's to obtain a sufficient precision $p$ that is essentially half of that of Section 3.3. This will have the effect of taking the square root of the decryption circuit degree.

\subsection{Using Less Precision}

We first sum the $s_{k}\left[\boldsymbol{t}_{k} \times \boldsymbol{\psi}\right]_{0}$ 's for $k<\gamma_{s e t}$, since they are iid, and then we add the remaining $c_{\gamma_{s e t}}$. The first sum will be represented on 6 bits ( 1 bit before the point and 5 bits after) and we will ensure that it is within $1 / 16$ of $\sum_{k<\gamma_{s e t}} s_{k}\left[\boldsymbol{t}_{k} \times\right.$ $\boldsymbol{\psi}]_{0} \bmod 2$, with high probability. We take $c_{\gamma_{s e t}}$ within distance $1 / 16$ of $\left[\boldsymbol{t}_{\gamma_{s e t}-1} \times\right.$ $\boldsymbol{\psi}]_{0} \bmod 2$ and represent it on 6 bits. The last sum will provide a result within distance $1 / 8$ of $\sum_{k \leq \gamma_{s e t}} s_{k}\left[\boldsymbol{t}_{k} \times \boldsymbol{\psi}\right]_{0} \bmod 2$, and can be done with a circuit of constant degree. Using Lemma 3.1, we obtain that the result is within $1 / 4$ of an integer.

We now concentrate on the first sum. Let the $c_{k}$ 's be fixed-point approximations to the $\left[\boldsymbol{t}_{k} \times \boldsymbol{\psi}\right]_{0}$ 's, with some precision $p$. We have $\varepsilon_{k} \leq 2^{-p}$ with $\varepsilon_{k}=$ $c_{k}-\left[\boldsymbol{t}_{k} \times \boldsymbol{\psi}\right]_{0}$. As the $c_{k}$ 's for $k<\gamma_{s e t}$ are iid, so are the $\varepsilon_{k}$ 's, $k<\gamma_{s e t}$. Also, 
we will ensure that $E\left[\varepsilon_{k}\right]=0$ for any $k<\gamma_{s e t}$. The following lemma leads to a probabilistic error bound for the sum of the $c_{k}$ 's.

Lemma 5.1. Let $\varepsilon_{1}, \ldots, \varepsilon_{t}$ be iid variables with values in $[-\varepsilon, \varepsilon]$ and such that $E\left[\varepsilon_{k}\right]=0$ for all $k$. Then $\left|\sum_{k \leq t} \varepsilon_{k}\right|>\sqrt{t} \varepsilon \cdot \omega(\sqrt{\log \lambda})$ with probability negligibly small with respect to $\lambda$.

Proof. We apply Hoeffding's inequality to the $\varepsilon_{k}$ 's. We have $\operatorname{Pr}\left[\left|\sum \varepsilon_{k}\right| \geq x\right] \leq$ $\exp \left(-x^{2} /\left(2 t \varepsilon^{2}\right)\right)$, for any $x>0$. We take $x=\sqrt{t} \varepsilon \cdot \omega(\sqrt{\log \lambda})$.

We use this lemma with $\varepsilon=2^{-p}$ and $t=\gamma_{s u b}-1$ (i.e., the number of non-zero $s_{k} \varepsilon_{k}$ 's for $\left.k<\gamma_{s u b}\right)$. It indicates that taking $p=\frac{1}{2} \log _{2} \gamma_{s u b}+$ $\omega(\log \log \lambda)$ suffices to ensure that with probability negligibly close to 1 we have $\left|\sum_{k<\gamma_{\text {set }}} s_{k}\left(c_{k}-\left[\boldsymbol{t}_{k} \times \boldsymbol{\psi}\right]_{0}\right) \bmod 2\right| \leq 1 / 32$. Truncating the result to 5 bits after the binary point cannot add more than an error of $1 / 32$.

\subsection{Expliciting the Computation of the $c_{k}$ 's in Enc"}

In order to be able to apply Lemma [5.1, we have to ensure that $E\left[\varepsilon_{k}\right]=0$ for any $k<\gamma_{\text {set }}$. To guarantee the latter and that this computation enjoys a limited complexity bound, the $c_{k}$ 's need to be computed carefully.

We are given $\boldsymbol{t}_{k}$ and $\boldsymbol{\psi}$, and wish to compute a $(1+p)$-bit approximation $c_{k}$ to $\left[\boldsymbol{t}_{k} \times \boldsymbol{\psi}\right]_{0} \bmod 2$. As $J$ is a degree 1 prime ideal, vector $\boldsymbol{\psi}$ is in fact an integer modulo $\operatorname{det}(J)$. We are thus interested in computing $\left[\boldsymbol{t}_{k}\right]_{0} \cdot \psi$ modulo 2 . We explicit this computation in Figure 4.

Inputs: Vectors $\boldsymbol{t}_{k}$ and $\boldsymbol{\psi}$, and precision $p$.

Output: A precision $(1+p)$ real $c_{k} \in[-1,1]$ with $\left|c_{k}-\left(\left[\boldsymbol{t}_{k} \times \boldsymbol{\psi}\right]_{0} \bmod 2\right)\right| \leq 2^{-p}$.

1. $p^{\prime}:=\log _{2} \operatorname{det}(J)+p+1$;

2. Compute the closest precision $\left(1+p^{\prime}\right)$ number $\bar{t}_{k} \in[-1,1]$ to $\left[\boldsymbol{t}_{k}\right]_{0}$.

3. Compute $c_{k}^{\prime}:=\bar{t}_{k} \psi$ exactly.

4. Reduce $c_{k}^{\prime}$ modulo 2, while preserving its sign (the result belongs to $[-1,1]$ ).

5 . Round $c_{k}^{\prime}$ to the closest precision $(1+p)$ number $c_{k} \in[-1,1]$.

Fig. 4. Computing coefficient $c_{k}$ for algorithm Enc ${ }^{\prime \prime}$

Lemma 5.2. The algorithm of Figure 4 is correct. Furthermore, if the vector $\boldsymbol{t}_{k}$ is chosen uniformly in $J^{-1} \bmod 2$ with uniformly random choice of sign when a coordinate of $\boldsymbol{t}_{k}$ belongs to $\{-1,1\}$, then $E\left[\varepsilon_{k}\right]=0$, where $\varepsilon_{k}=c_{k}-\left(\left[\boldsymbol{t}_{k} \times\right.\right.$ $\left.\psi]_{0} \bmod 2\right)$.

Proof. At Step 2 of the algorithm, we have $\left|\bar{t}_{k}-\left[\boldsymbol{t}_{k}\right]_{0}\right| \leq 2^{-p^{\prime}-1}$. As $\psi$ is exact and belongs to $[0, \operatorname{det} J)$, we have $\left|\bar{t}_{k} \psi-\left[\boldsymbol{t}_{k}\right]_{0} \psi\right| \leq 2^{-p^{\prime}-1} \operatorname{det}(J) \leq 2^{-p-1}$. Thus, at Step 3, we have $\left|c_{k}^{\prime}-\left[\boldsymbol{t}_{k} \times \boldsymbol{\psi}\right]_{0}\right| \leq 2^{-p-1}$. The rounding of Step 5 leads to $\left|c_{k}-\left(\left[\boldsymbol{t}_{k} \times \boldsymbol{\psi}\right]_{0} \bmod 2\right)\right| \leq 2^{-p-1}+2^{-p-1}=2^{-p}$. 
To prove the second statement, we use the symmetry of the distribution of $\boldsymbol{t}_{k}$. It implies that $E\left[\left[\boldsymbol{t}_{k} \times \boldsymbol{\psi}\right]_{0} \bmod 2\right]=0$. We now use the same property to show that $E\left[c_{k}\right]=0$. At Step 2, changing $\boldsymbol{t}_{k}$ into $-\boldsymbol{t}_{k}$ has the effect of changing $\bar{t}_{k}$ into $-\bar{t}_{k}$. This implies that at Step 3, changing $\boldsymbol{t}_{k}$ into $-\boldsymbol{t}_{k}$ has the effect of changing $c_{k}^{\prime}$ into $-c_{k}^{\prime}$. Due to the symmetry of the rounding to nearest, this carries over to $c_{k}$ and $\varepsilon_{k}$ at Step 5 .

Note that the choice of rounding to nearest is not benign: the above proof strongly relies on the symmetry of the rounding with respect to 0 .

\subsection{Decreasing the Decryption Circuit Depth}

We now want to compute $\sum_{k<\gamma_{s e t}} s_{k} c_{k} \bmod 2$, where the $c_{k}$ 's are fixed-point reals with precision $p=\frac{1}{2} \log _{2} \gamma_{s u b}+\omega(\log \log \lambda)$. Instead of computing the Hamming weights $W_{j}$ for $j \in\{0, \ldots, p\}$ as in the proof of Theorem 3.1, we compute only the bits $W_{j, \ell}$ (for $0 \leq \ell \leq\left\lceil\log _{2} \gamma_{s u b}\right\rceil$ ) that are going to contribute to $\sum_{k<\gamma_{s e t}} s_{k} c_{k} \bmod 2$ : the most significant bits are rendered useless by the reduction modulo 2. Most interestingly, these unnecessary most significant bits were the ones requiring the higher degree circuits to evaluate. More precisely, we have:

$$
\sum_{k<\gamma_{s e t}} s_{k} c_{k}=\sum_{j=0}^{p} \sum_{\ell=0}^{\left\lceil\log _{2} \gamma_{s u b}\right\rceil} W_{j, \ell} 2^{-j+\ell}=\sum_{j=0}^{p} \sum_{\ell=0}^{j+1} W_{j, \ell} 2^{-j+\ell} \bmod 2 .
$$

Lemma 3.4 now implies that the desired sum mod 2 can be computed correctly with probability negligibly close to 1 with respect to $\lambda$, by evaluating an arithmetic circuit of size $\mathcal{P}$ oly $\left(\gamma_{s u b}\right)$ corresponding to a polynomial of degree exactly $2^{p+1}=\sqrt{\gamma_{s u b}} \cdot \omega(\sqrt{\log \lambda})$. Overall, we get:

Theorem 5.1. The scheme SqHom is bootstrappable as long as

$$
\sqrt{\gamma_{s u b}} \cdot \omega(\sqrt{\log \lambda}) \leq \frac{\log r_{D e c}^{\prime}}{\log \left(r_{E n c} \cdot \gamma_{\times} \cdot(t+1)\right)} .
$$

\section{Asymptotic Efficiency}

We now use the improvements described in the two previous sections to derive bounds for the complexity of Gentry's fully homomorphic scheme.

\subsection{Optimizing the Parameters in Gentry's Scheme}

The table below summarizes and compares the conditions for Gentry's scheme to be $2^{\lambda}$-secure and correct. The semantic security of SomHom' is related to the hardness of $\gamma$-BDD for $\gamma=r_{D e c}^{\prime} / r_{E n c}$. Recall that $r_{D e c}^{\prime}=\lambda_{1}(J) / \mathcal{P}$ oly $(n)$. Recall also that $J$ is an ideal lattice, and thus we have $\lambda_{1}(J) \geq \operatorname{det}(J)^{1 / n}=q^{1 / n} / 2$ (where $q$ is the SVSSP determinant of Section 4). As a consequence, it suffices 
to ensure that $\gamma$-BDD is hard to solve for $\gamma=q^{1 / n} /\left(r_{E n c} \mathcal{P}\right.$ oly $\left.(n)\right)$. We use the lattice reduction 'rule of thumb' to derive a sufficient condition. As the encryptor is limited to polynomial-time algorithms, we can safely assume that $n=\mathcal{P}$ oly $(\lambda)$. Also, since $f=x^{n}+1$, we have $\gamma_{\times}=\sqrt{n}$. Finally, by choosing $r_{E n c}=\mathcal{P} \operatorname{ly}(\lambda)$, the ciphertexts have sufficient entropy to prevent any exhaustive search.

\begin{tabular}{|c|r|c|}
\hline Condition & {$[9]$} & This article \\
\hline BDD resistant to lattice attacks & \multicolumn{2}{|c|}{$\frac{q^{1 / n}}{\mathcal{P} \text { oly }(\lambda)} \leq c_{1}^{n / \lambda}$} \\
\hline SSSP resistant to birthday paradox & \multicolumn{2}{|c|}{$\left(\begin{array}{c}\gamma_{s e t} \\
\gamma_{s u b}\end{array}\right)^{1 / 2} \geq 2^{\lambda}$} \\
\hline SSSP resistant to lattice attacks & $\gamma_{\text {set }}=\widetilde{\Omega}(\log q)$ & $\frac{\gamma_{s e t}^{2}}{\lambda}=\widetilde{\Omega}(\log q)$ \\
\hline Bootstrappability achieved & $\gamma_{s u b} \leq \frac{\log \left(q^{1 / n}\right)}{\Theta(\log \lambda)}$ & $\sqrt{\gamma_{s u b}} \leq \frac{\log \left(q^{1 / n}\right)}{\mathcal{P} \text { oly }(\log \lambda)}$ \\
\hline
\end{tabular}

To fulfill these conditions, we set $\gamma_{s u b}=\Theta(\lambda), n=\widetilde{\Theta}\left(\lambda^{1.5}\right), \log q=\widetilde{\Theta}\left(\lambda^{2}\right)$ and $\gamma_{\text {set }}=\Theta\left(\lambda^{1.5}\right)$. In [8, Ch. 12], these values were $\gamma_{s u b} \approx \lambda, n \approx \lambda^{2}, \log q \approx \lambda^{3}$ and $\gamma_{\text {set }} \approx \lambda^{3}$ respectively.

\subsection{Bit Complexity}

The Recrypt procedure consists in expanding the ciphertext $\psi$ as described in algorithm Enc" of SqHom, encrypting the bits of the expanded ciphertext with the new public key $p k_{2}$, and then applying algorithm Dec" homomorphically, using the encrypted ciphertext bits and the encrypted secret key $s k_{1}$ (under $p k_{2}$ ). We also consider the cost of homomorphically evaluating an elementary add/mult gate.

Let us first bound the cost of computing the $c_{k}$ 's in Enc', calling $\gamma_{\text {set }}$ times the algorithm from Figure 4. First, note that Steps 1 and 2 should not be done within Enc", but at the key generation time, i.e., in KeyGen". Note that during the third step of KeyGen", one should also pay attention to perform the reduction modulo (2) such that the assumption of Lemma 5.2 holds. The quantity $c_{k}^{\prime}$ obtained at Step 3 of the algorithm from Figure 4 is encoded on $O(\log q)$ bits, and its computation can be performed in $\widetilde{O}(\log q)$ bit operations, using fast integer arithmetic 25. The costs of Steps 4 and 5 are negligible. Overall, the computation of the $c_{k}$ 's in Enc' can be done in $\widetilde{O}\left(\gamma_{\text {set }} \log q\right)=\widetilde{O}\left(\lambda^{3.5}\right)$ bit operations.

The secret key is made of $\gamma_{s e t}=\Theta\left(\lambda^{1.5}\right)$ bits. The bit-length of the encrypted secret key is $\gamma_{\text {set }} \log q=\widetilde{O}\left(\lambda^{3.5}\right)$. To encrypt the bits of the $c_{k}$ 's under $p k_{2}$, we use Samp $=0$, as explained in [8, Re. 4.1.1], i.e., we consider as encrypted values the bits themselves.

Let us now explain how algorithm Dec" is implemented. We concentrate on the most expensive part, i.e., the (homomorphic) computations of $O\left(\log \gamma_{s u b}\right)=$ $\widetilde{O}(1)$ Hamming weights of vectors in $\{0,1\} \gamma^{\gamma_{s e t}}$. Let $\left(\alpha_{1}, \ldots, \alpha_{\gamma_{s e t}}\right)$ be such a vector. As explained in [9, Le. 5] (which relies on [2, Le. 11]), it suffices to compute the developed form of the polynomial $\prod_{k \leq \gamma_{s e t}}\left(x-\alpha_{k}\right)$. Recall that in Section 5 we showed that we are interested in only a few coefficients of the result, corresponding to monomials of degrees $\widetilde{O}\left(\sqrt{\gamma_{s u b}}\right)$. For the sake of simplicity (and with a negligible cost increase), we compute the full developed form anyway, and then throw away the spurious coefficients. Our circuit here differs from those of [26 5] and [8, Ch. 9] 
as we use fast polynomial multiplications and a tree-based construction instead of school-book multiplications and Horner's method, to lower the overall asymptotic complexity. Note that the circuit is over the integers, and evaluates an integer polynomial whose coefficients of interest have small multiplicative degrees in the inputs. We compute the developed form of $\prod_{k \leq \gamma_{s e t}}\left(x-\alpha_{k}\right)$ with a binary tree:

- At level 0 , we have the linear factors $\left(x-\alpha_{k}\right)$.

- At level $i$, we have $\gamma_{\text {set }} / 2^{i}$ polynomials of degree $2^{i}$ that are the products of the linear factors corresponding to their binary subtrees.

- A father of two nodes is obtained by multiplying his two sons, with a quasilinear time multiplication for polynomials over rings that uses only ring operations [3].

The size of each circuit that allows to move from sons at level $i-1$ to father at level $i$ is $\widetilde{O}\left(2^{i}\right)$. The overall number of add/mult integer gates is therefore $\widetilde{O}\left(\gamma_{s e t}\right)$. While evaluating this circuit homomorphically, each gate corresponds to an add/mult modulo $B_{J}^{p k}$, i.e., thanks to our choice for $J$, to an add $/$ mult of two integers modulo $\operatorname{det}(J)$, whose bit-length is $O(\log q)$. The overall complexity of $\operatorname{Dec}^{\prime \prime}$ is $\widetilde{O}\left(\gamma_{s e t} \log q\right)=\widetilde{O}\left(\lambda^{3.5}\right)$.

To summarize, Recrypt for 1 plaintext bit costs $\widetilde{O}\left(\lambda^{3.5}\right)$ bit operations (compared to the bound $\widetilde{O}\left(\lambda^{6}\right)$ claimed in [8, Ch. 12]). And the cost of homomorphically evaluating an elementary add/mult gate is also $\widetilde{O}\left(\lambda^{3.5}\right)$. The secret $s$ and the public key $\left(B_{J}^{p k} ; \overline{\boldsymbol{t}}_{1}, \ldots, \overline{\boldsymbol{t}}_{\gamma_{s e t}}\right)$ are respectively encoded on $\gamma_{s e t}=\Theta\left(\lambda^{1.5}\right)$ and $\widetilde{O}\left(n \log q+\gamma_{\text {set }} \log q\right)=\widetilde{O}\left(\lambda^{3.5}\right)$ bits.

\section{Open Problems}

It would be interesting to relax our assumptions $f=x^{n}+1$ and $I=(2)$, in case other choices prove interesting (see the full version for $I=(2, x+1)$ ). An important question is to assess the practical impact of our results (see [26]12] for implementations of Gentry's scheme). At the end of [8, Se. 12.3], Gentry suggests using non-independent SplitKey vectors $\boldsymbol{t}_{i}$ to lower the costs. The idea is to encode $n$ vectors $\boldsymbol{t}_{i, j}=x^{j} \boldsymbol{t}_{i} \bmod x^{n}+1$ using only $\boldsymbol{t}_{i}$. This leads to a faster amortized cost per plaintext bit using the plaintext domain $\mathbb{Z}_{2}[x] / f(x)$. However, it is not clear how to homomorphically decrypt with such a variant, as one is now restricted to more complex circuit gates than addition and multiplication modulo 2.

Acknowledgments. We thank Ali Akhavi, Guillaume Hanrot, Steven Galbraith, Craig Gentry and Paul Zimmermann for helpful discussions. We also thank the anonymous reviewers, for pointing out an important error in Section 4. The first author was partly supported by the LaRedA ANR grant, and the second author by a Macquarie University Research Fellowship (MQRF) and ARC Discovery Grant DP0987734.

\section{References}

1. Babai, L.: On Lovász' lattice reduction and the nearest lattice point problem. Combinatorica 6, 1-13 (1986) 
2. Boyar, J., Peralta, R., Pochuev, D.: On the multiplicative complexity of boolean functions over the basis $(\wedge, \oplus, 1)$. Theoret. Comput. Sci. 235(1), 43-57 (2000)

3. Cantor, D.G., Kaltofen, E.: On fast multiplication of polynomials over arbitrary algebras. Acta Inf. 28(7), 693-701 (1991)

4. Cassels, J.W.S.: An Introduction to the Geometry of Numbers, 2nd edn. Springer, Heidelberg (1971)

5. van Dijk, M., Gentry, C., Halevi, S., Vaikuntanathan, V.: Fully homomorphic encryption over the integers. In: Gilbert, H. (ed.) EUROCRYPT 2010. LNCS, vol. 6110, pp. 24-43. Springer, Heidelberg (2010)

6. Gama, N., Nguyen, P.Q.: Finding short lattice vectors within Mordell's inequality. In: Proc. of STOC, pp. 207-216. ACM, New York (2008)

7. Gama, N., Nguyen, P.Q.: Predicting lattice reduction. In: Smart, N.P. (ed.) EUROCRYPT 2008. LNCS, vol. 4965, pp. 31-51. Springer, Heidelberg (2008)

8. Gentry, C.: A fully homomorphic encryption scheme. PhD thesis, Stanford University (2009), http://crypto.stanford.edu/craig (manuscript)

9. Gentry, C.: Fully homomorphic encryption using ideal lattices. In: Proc. of STOC, pp. 169-178. ACM, New York (2009)

10. Gentry, C.: Computing arbitrary functions of encrypted data. Communications of the ACM 53(3), 97-105 (2010)

11. Gentry, C.: Toward basing fully homomorphic encryption on worst-case hardness. In: Rabin, T. (ed.) CRYPTO 2010. LNCS, vol. 6223, pp. 116-137. Springer, Heidelberg (2010)

12. Gentry, C., Halevi, S.: Implementing Gentry's fully-homomorphic encryption scheme. Preliminary version (August 5, 2010),

https://researcher.ibm.com/researcher/files/us-shaih/ fhe-implementation.pdf

13. Hoeffding, W.: Probability inequalities for sums of bounded random variables. J. Amer. Statist. Assoc. 58(301), 13-30 (1963)

14. Kannan, R.: Improved algorithms for integer programming and related lattice problems. In: Proc. of STOC, pp. 99-108. ACM, New York (1983)

15. Karp, R.M.: A survey of parallel algorithms for shared-memory machines. Technical report (1988)

16. Klein, P.N.: Finding the closest lattice vector when it's unusually close. In: Proc. of SODA, pp. 937-941. ACM, New York (2000)

17. Lenstra, A.K., Lenstra Jr., H.W., Lovász, L.: Factoring polynomials with rational coefficients. Math. Ann. 261, 515-534 (1982)

18. Ludwig, C.: A faster lattice reduction method using quantum search. In: Ibaraki, T., Katoh, N., Ono, H. (eds.) ISAAC 2003. LNCS, vol. 2906, pp. 199-208. Springer, Heidelberg (2003)

19. Micciancio, D.: Improving lattice-based cryptosystems using the Hermite normal form. In: Silverman, J.H. (ed.) CALC 2001. LNCS, vol. 2146, pp. 126-145. Springer, Heidelberg (2001)

20. Micciancio, D., Goldwasser, S.: Complexity of lattice problems: a cryptographic perspective. Kluwer Academic Press, Dordrecht (2002)

21. Micciancio, D., Regev, O.: Lattice-based Cryptography. In: Post-Quantum Cryptography. Springer, Heidelberg (2008)

22. Micciancio, D., Voulgaris, P.: A deterministic single exponential time algorithm for most lattice problems based on Voronoi cell computations. In: Proc. of STOC, pp. 351-358. ACM, New York (2010) 
23. Nguyen, P.Q., Shparlinski, I.: On the insecurity of a server-aided RSA protocol. In: Boyd, C. (ed.) ASIACRYPT 2001. LNCS, vol. 2248, pp. 21-35. Springer, Heidelberg (2001)

24. Schnorr, C.P.: A hierarchy of polynomial lattice basis reduction algorithms. Theoret. Comput. Sci. 53, 201-224 (1987)

25. Schönhage, A., Strassen, V.: Schnelle Multiplikation grosser Zahlen. Computing 7, 281-292 (1971)

26. Smart, N.P., Vercauteren, F.: Fully homomorphic encryption with relatively small key and ciphertext sizes. In: Nguyen, P.Q., Pointcheval, D. (eds.) PKC 2010. LNCS, vol. 6056, pp. 420-443. Springer, Heidelberg (2010)

\section{A Smaller Keys}

In [8, Se. 4.3], Gentry suggests to re-use the same key-pair for all levels of the fully homomorphic scheme derived from Theorem 2.2. This allows one to significantly decrease the key-sizes of the boostrapped fully homomorphic scheme. This strategy can be proved secure if the underlying bootstrappable homomorphic encryption scheme is assumed or known to be KDM-secure [8, Th. 4.3.2]. Our lower-degree decryption may fail with non-negligible probability after the first refreshing of a ciphertext, as our technique does not handle the non-independence of the ciphertext and the secret key. To circumvent this issue, we randomize the ciphertext to waive its possible non-independence with the secret key. Note that this technique is similar in flavor to Gentry's modified scheme providing circuit privacy [9, Se. 7].

Consider algorithm Enc" of SqHom. The condition required for the probabilistic technique described in Section 5 to work is that the ciphertext $\boldsymbol{\psi}=$ $\pi+\boldsymbol{r} \bmod B_{J}^{p k}$ (where $\boldsymbol{r} \in(2)$ and $\|\boldsymbol{r}\| \leq r_{D e c}^{\prime}$ ) is independent of the $\boldsymbol{t}_{i}$ 's. This fact, together with the iid-ness of the $\boldsymbol{t}_{i}$ 's, implies that the rounding errors $\varepsilon_{i}$ in computing the $c_{i}$ 's, are iid, as required to apply Hoeffding's bound. In the key-reuse application, the internal randomness $\boldsymbol{r}$ of $\boldsymbol{\psi}$ may depend on the $\boldsymbol{t}_{i}$ 's (due to a previous refreshing). To circumvent this, we randomize the ciphertext $\boldsymbol{\psi}=\pi+\boldsymbol{r} \bmod B_{J}^{p k}$ into another ciphertext $\boldsymbol{\psi}^{\prime}=\pi+\boldsymbol{r}^{\prime} \bmod B_{J}^{p k}$ for the same message $\pi$ but with internal randomness $\boldsymbol{r}^{\prime} \in(2)$ which is almost independent of the $\boldsymbol{t}_{i}$ 's. More precisely, given the $\boldsymbol{t}_{i}$ 's, the distribution of $\boldsymbol{r}^{\prime}$ is within negligible statistical distance from the $\left(\boldsymbol{t}_{i}\right.$-independent) distribution $2 U$, where $U$ is the uniform distribution on the origin-centered ball of radius $r_{D e c}^{\prime} / \rho$ with $\rho$ any negligible function of $\lambda$ such that $\log \rho=\widetilde{O}(1)\left(\right.$ e.g., $\left.\rho=\lambda^{-\log \lambda}\right)$.

We compute $\boldsymbol{\psi}^{\prime}$ by adding to $\boldsymbol{\psi}$ an encryption of 0 with sufficiently large randomness compared to the randomness in $\boldsymbol{\psi}$, i.e., we set $\boldsymbol{\psi}^{\prime}=\boldsymbol{\psi}+\boldsymbol{\zeta} \bmod B_{J}^{p k}$, where $\zeta$ is sampled from $2 U$. If we replace the decryption radius $r_{D e c}^{\prime}$ by $r_{D e c}^{\prime \prime}=\frac{r_{D e c}^{\prime}}{1+2 / \rho}$ in Lemma 3.2, then the correctness of the scheme is preserved, as $\boldsymbol{\psi}$ and $\boldsymbol{\psi}^{\prime}$ both decode to the same plaintext via algorithm $\mathrm{Dec}^{\prime}$. This has a negligible effect for the asymptotic efficiency (see Section 6.1). Assume that $\boldsymbol{\psi}=\pi+\boldsymbol{r} \bmod B_{J}^{p k}$ with $\|\boldsymbol{r}\| \leq r_{D e c}^{\prime}$. Let us consider the statistical distance between the distributions $\boldsymbol{r}+2 U$ and $2 U$. As a ball of radius $r_{D e c}^{\prime} / \rho-r_{D e c}^{\prime}$ is contained in the intersection of the two balls of radius $r_{D e c}^{\prime} / \rho$ corresponding to $U$ and $\boldsymbol{r}+U$, we obtain that the statistical distance under scope is at most $n \cdot \rho$, and hence negligible. 